\title{
Beyond the Quintessential Quincunx
}

\author{
Michael A. Proschan ${ }^{1}$ and Jeffrey S. Rosenthal ${ }^{2}$
}

\begin{abstract}
The quincunx, a contraption with balls rolling through a triangle-shaped arrangement of nails, was invented to illustrate the binomial distribution and the central limit theorem for Bernoulli random variables. As it turns out, a modification of the quincunx can be used to teach many different concepts, including the central limit theorem for independent but not identically distributed random variables, permutation tests in a paired setting, and the generation of a random variable with an arbitrary continuous distribution from a uniform variate. This paper uses a universal quincunx-"uncunx"-applet to illustrate these and other applications.
\end{abstract}

KEY WORDS: Bernoulli Random Variables; Binomial Distribution; Central Limit Theorem; Permutation Test; Random Number Generation; Symmetric Distribution

\section{Introduction}

The Quincunx is a device invented by Francis Galton to illustrate the binomial distribution and the central limit theorem (CLT) for Bernoulli random variables. For the very interesting history, see pages 275-281 of Stigler (1986) or Kunert, Montag, and Pohlmann (2001). In this device, balls roll one-by-one down a board, striking nails head-on and deflecting to the left or right, and then collect in bins at the bottom (Figure 1).

Each deflection is a binary random variable parameterized with left and right corresponding to -1 and +1 , respectively. Regardless of the deflections in previous rows, the ball is equally likely to go left or right in the current and future rows, so the deflections in different rows are independent. The position of the ball at the end is determined by the sum of the deflections; with $2 n$ rows, if there are just as many left as right deflections, the sum of the $-1 \mathrm{~s}$ and $+1 \mathrm{~s}$ is $S_{2 n}=0$ and the ball will end in the middle chute. On the other hand, if all of the deflections are to the right $(+1)$, the sum will be $S_{2 n}=2 n$ and the ball will end in the rightmost chute, etc. Each ball is a single realization of $S_{2 n}$, whereas the proportions of balls in the different chutes represent the simulated distribution of $S_{2 n}$. With a large number of balls, the simulated distribution will tend to the true distribution of $S_{2 n}$. If we relabel left and right deflections as 0 and 1 instead of -1 and +1 , the distribution of $S_{2 n}$ is binomial

\footnotetext{
${ }^{1}$ National Institute of Allergy and Infectious Diseases (E-mail: ProschaM@mail.nih.gov)

${ }^{2}$ University of Toronto (E-mail: jeff@math.toronto.edu)
} 


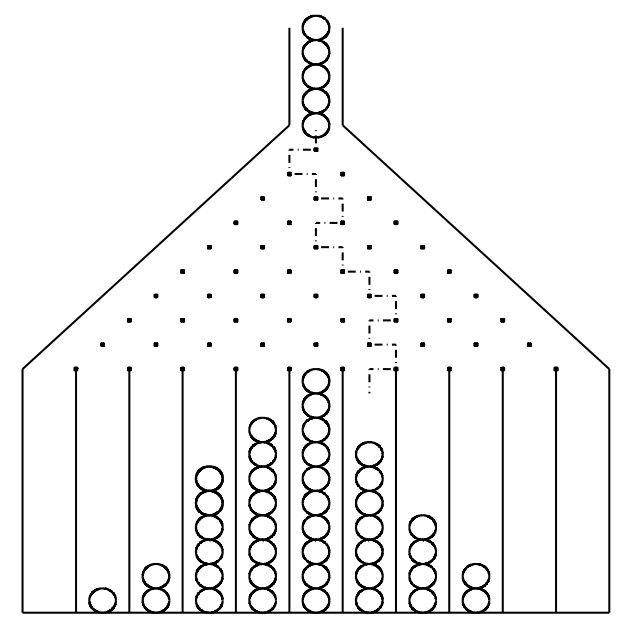

Figure 1: A quincunx. Balls roll down the board, striking one nail in each row and bouncing to the left or right with equal probability. The bell-shaped distribution of balls in the bins at the bottom simulates the binomial distribution and illustrates the CLT.

$(2 n, 1 / 2)$. With a large number of rows, this binomial distribution will look bell-shaped, consistent with the CLT. Of course the quincunx shows only that some bell-shaped distribution applies, not necessarily the normal. For a heuristic justification of why only a normal distribution fits, see Proschan (2007).

Commercial quincunxes are scarce and expensive, and building even a simple one (Figure 2 ) is a painstaking endeavor because the nails must be placed in just the right places, the board must be very straight, and the balls must be the same size and not have imperfections. Building a universal quincunx that incorporates the modifications we make in this paper is nearly impossible. Fortunately, a universal quincunx - "uncunx"- applet is available at

$$
\text { http : //probability.ca/jeff/java/uncunx.html }
$$

The distinguishing feature of an uncunx is that the deflection sizes may differ from row to row, and even from left to right within a row. This means that the number of nails depends on the deflection sizes; with equal deflection sizes, the number of nails in row $i$ is $i$, but with unequal deflection sizes it can be up to $2^{i-1}$. This can quickly clutter an uncunx. The uncunx applet has 9 rows and shows triangles instead of nails. To avoid the clutter problem just alluded to, it shows the triangles only when the balls are actually branching. Clicking 
on the display and typing "f" or "s" causes the balls to go faster or slower, respectively. As each ball reaches the bottom of the uncunx, the corresponding histogram bar becomes taller. Also shown is a normal curve with the same mean and variance as the binomial. As more balls accumulate, the histogram looks very similar to the normal curve. Later we will illustrate more sophisticated features of the applet.

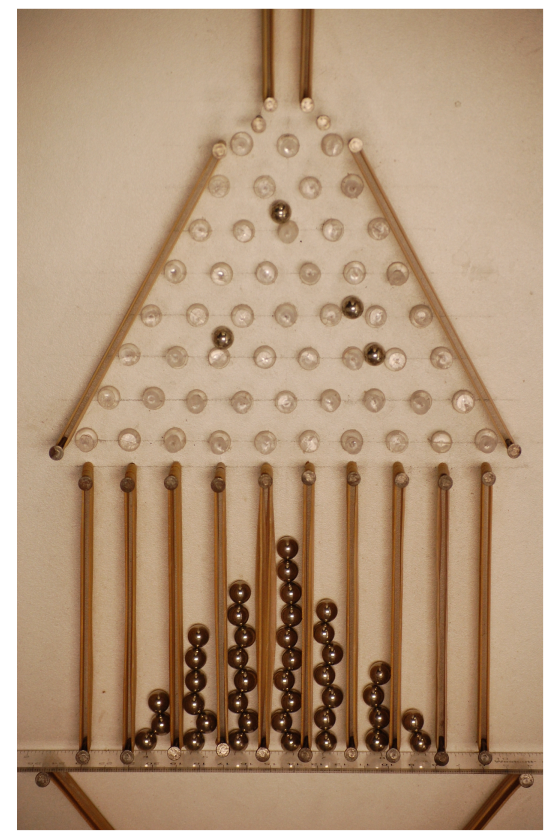

Figure 2: A homemade quincunx made with pushpins, rubber bands, a ruler, steel balls, nails, and a board.

This paper argues that the uncunx applet is a wonderful teaching device that can be used to illustrate many different concepts in probability and statistics, including Pascal's triangle, CLTs for independent but not identically distributed random variables, and permutation tests. The presentation is heuristic rather than rigorous, but the intuition instilled by the uncunx applet is valuable for statistics students of all levels.

\section{Pascal's Triangle}

One reason a quincunx is helpful is that it gets students to think about the number of paths a ball can take, which can help with combinatorics. For this application only, it is helpful to number the rows sequentially beginning with 0 instead of the more conventional 1. Similarly, number the nails in each row sequentially beginning with nail 0 . How many paths lead to nail $k$ of row $r$ of the quincunx? Denote this number by $N_{r, k}$. The number of paths is simple 


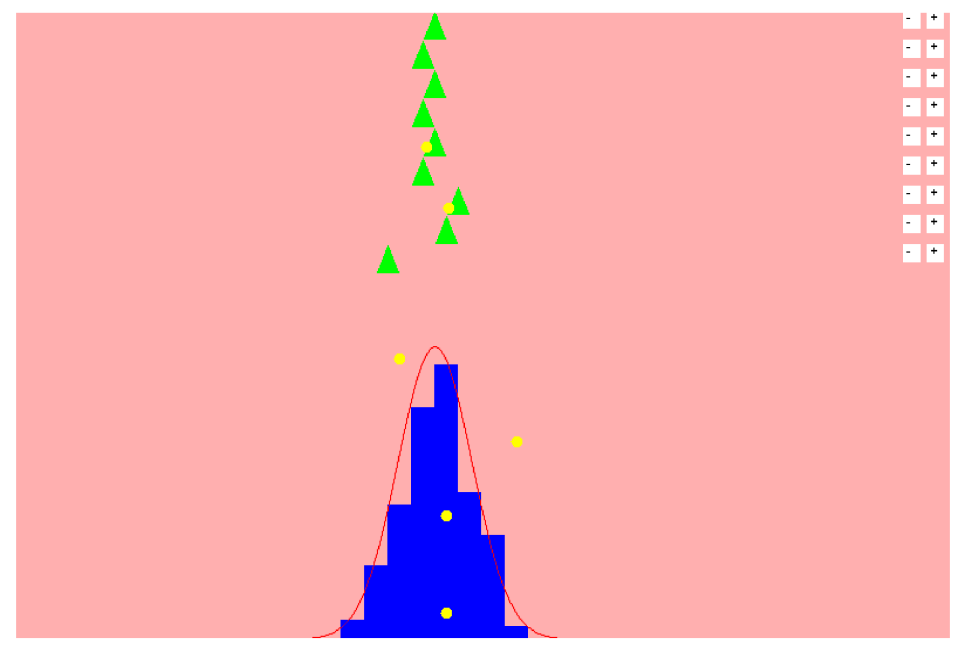

Figure 3: The uncunx applet in its default mode with equal row deflections. The superimposed normal distribution has the same mean and variance as the binomial it simulates.

if $k=0$; every deflection must be to the left, so $N_{r, 0}=1$. Similarly, $N_{r, r}=1$. Now consider nail $k$ of row $r$, where $k \neq 0$ and $k \neq r$. For the ball to strike nail $k$ in row $r$, in row $r-1$ it must have struck either nail $k-1$ and bounced right, or nail $k$ and bounced left. Therefore, the number of paths leading to nail $k$ in row $r$ is the sum of the numbers of paths striking nails $k-1$ and $k$ of row $r-1$. In symbols, $N_{r, k}=N_{r-1, k-1}+N_{r-1, k}$ (see equation 1.11 on page 37 of Parzen, 1960). This suggests an iterative way to determine $N_{r, k}$. Place a 1 in row 0 and at the extreme left and right ends of each row. To get the number corresponding to a given nail in a row, add the numbers in the preceding row just to the left and just to the right of the position in the current row. This is the algorithm for Pascal's triangle (Table 1). The equation $N_{r, k}=N_{r-1, k-1}+N_{r-1, k}$ is usually proven by induction, which offers no intuition. The quincunx is a concrete model that provides the intuition.

\section{Central Limit Theorems for Symmetric Binary Ran- dom Variables}

The Introduction explained that the bell-shaped distribution of balls in the different chutes illustrates the CLT for iid symmetric Bernoulli random variables, but does the CLT hold for 
Table 1: Pascal's Triangle. Each element of a row other than the ones at the end is obtained by adding the two adjacent elements of the preceding row.

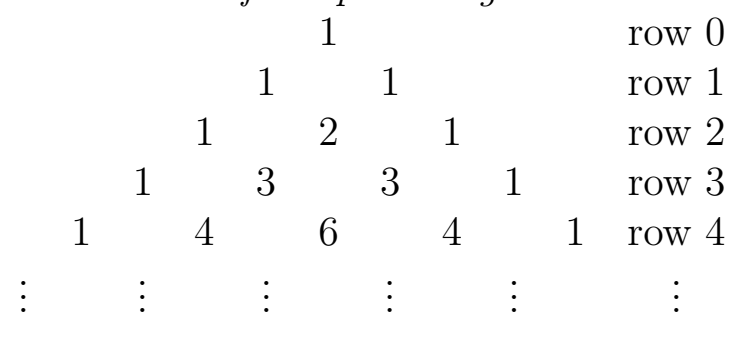

independent but not identically distributed symmetric binary random variables $D_{1}, \ldots, D_{n}$ ? Think of $D_{i}$ as the deflection in row $i$ of a quincunx with unequal deflection lengths $\left|d_{i}\right|, i=$ $1, \ldots, n$; then $D_{1}, \ldots, D_{n}$ are independent binary random variables with $\operatorname{Pr}\left(D_{i}=+\left|d_{i}\right|\right)=$ $\operatorname{Pr}\left(D_{i}=-\left|d_{i}\right|\right)=1 / 2$. Will the sum $S_{n}=\sum_{i=1}^{n} D_{i}$ still be approximately normal? The applet at the URL (1) can be used to answer this question. The + or - boxes in the upper right portion of the applet enlarge or shrink the deflections in the different rows. The variance of the superimposed normal distribution, $\sum d_{i}^{2}$, changes as we change the $\left|d_{i}\right|$. Click on the + box of the first row 15 times. The first deflection dominates all other rows, and the resulting bimodal histogram is clearly not approximately normal (Figure 4). The student can play with the deflection sizes to gain insight about how dissimilar they have to be for the resulting histogram to appear non-normal (but beware that it is possible to enlarge the deflections so much that the balls fall outside the display). From a practical standpoint, the applet simulation is more directly relevant than theoretical asymptotic results. After all, even if we can prove that $S_{n}$ is asymptotically normal under certain conditions, $n$ may need to be very large for the normal approximation to be accurate.

The bimodal distribution from the uncunx applet suggests a way to construct an infinite set of symmetric binary random variables such that $S_{n}$ is not asymptotically normal. We can make the deflection in the first row dwarf the sum of all subsequent deflections. To make it easy to compute the sum of all subsequent absolute deflections, choose $\left|d_{2}\right|,\left|d_{3}\right|,\left|d_{4}\right| \ldots$ to be a geometric series, say $\left|d_{2}\right|=1 / 4,\left|d_{3}\right|=1 / 8,\left|d_{4}\right|=1 / 16$ etc. Then $\sum_{i=2}^{\infty}\left|d_{i}\right|=$ $(1 / 4) /(1-1 / 2)=1 / 2$. Simply choose $\left|d_{1}\right|$ larger than $1 / 2$, say $\left|d_{1}\right|=1$. This will create two separate humps in the distribution of the sum similar to what happened in Figure 4. For example, if $D_{1}=-1$, then even if all of the remaining $D_{i}$ are positive, $S_{n} \leq-1+1 / 2=-1 / 2$. Similarly, if $D_{1}=+1$, then even if all of the remaining $D_{i}$ are negative, $S_{n} \geq 1-1 / 2=1 / 2$. 


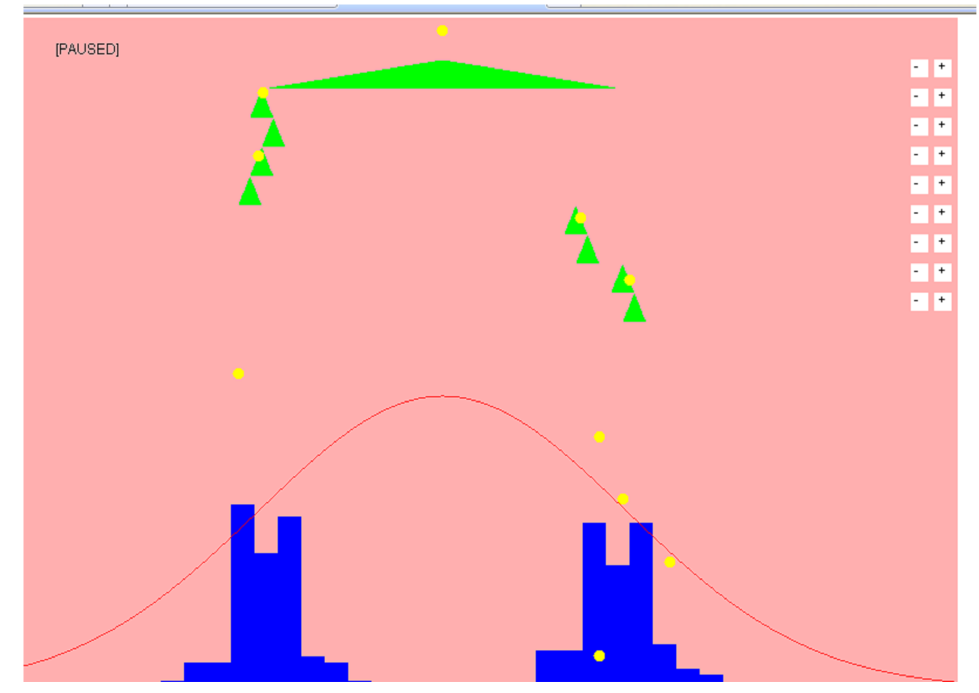

Figure 4: The applet after clicking on the "+" button in the first row 15 times to make its deflection dominate all others, causing a bimodal distribution.

Therefore, $P\left(-1 / 2<S_{n}<1 / 2\right)=0$ for all $n$, and the distribution of $S_{n}$ has two symmetric humps. The conditions ensuring asymptotic normality must therefore rule out cases like this in which one row's deflection dominates the overall sum. Students in undergraduate probability and statistics courses need not understand the precise Lindeberg condition that ensures normality (e.g., see page 239 of Feller, 1957), just that some condition is needed to prevent one row from dominating.

\section{Permutation Tests in A Paired Setting}

Sometimes data consist of pairs, one member of which is given a treatment $(\mathrm{T})$ and the other a control $(\mathrm{C})$. Examples are crossover studies or clinical trials with pair matching. Under the null hypothesis of no treatment effect, the distribution of paired differences should be symmetric about 0 . One attractive option for analyzing such data is a permutation test (Good, 2005). We condition on the absolute value of pairwise differences, $\left|d_{i}\right|, i=1, \ldots, n$ Conditioned on $\left|d_{i}\right|$, the $i$ th paired difference is equally likely to be $\pm\left|d_{i}\right|$, so the test statistic $\sum_{i=1}^{n} D_{i}$ is conditionally a sum of independent but not identically distributed binary random variables. The distribution of this sum is the permutation distribution. The situation is 
identical to that of the preceding section. We can use the uncunx applet to see whether, given $\left|d_{1}\right|, \ldots,\left|d_{n}\right|$, the permutation distribution is approximately normal.

Most statisticians have a vague notion that when the sample size is large, the permutation distribution is approximately normal, so the permutation test gives the same answer as the t-test asymptotically. But what does this statement really mean? We saw that it is possible to make up values $\left|d_{1}\right|, \ldots,\left|d_{n}\right|$ such that the permutation distribution is not approximately normal, even if $n$ is large. Are such sets of $\left|d_{1}\right|, \ldots,\left|d_{n}\right|$ aberrant, or could they reasonably arise as iid realizations from a distribution with finite variance? To investigate this, click on the uncunx display and press "d" for distribution. This causes the applet to choose row deflection sizes iid from a uniform distribution on $[1,40]$. Despite the fact that this distribution is very spread out and this uncunx has only 9 rows, the permutation distribution is nearly always approximately normal with mean 0 and variance $\sum_{i=1}^{n} d_{i}^{2}$. That is, $Z_{n}=S_{n} /\left(\sum_{i=1}^{n} d_{i}^{2}\right)^{1 / 2}$ is approximately standard normal for any values $\left|d_{1}\right|, \ldots,\left|d_{n}\right|$ that might arise as iid observations from this distribution. More generally, for any distribution of row deflection sizes with finite variance, the set of infinite realizations $\left|d_{1}\right|, \ldots,\left|d_{n}\right|, \ldots$ for which the permutation distribution of $Z_{n}=S_{n} /\left(\sum_{i=1}^{n} d_{i}^{2}\right)^{1 / 2}$ does not approach $\mathrm{N}(0,1)$ as $n \rightarrow \infty$ has probability 0 of occurring. In other words, we get the same answer asymptotically whether we use a t-test or a permutation test in the paired setting. Van der Vaart (1998) proves the analog of this result in an unpaired setting.

\section{The CLT for Symmetric Random Variables}

The arguments of the preceding section can be used to deduce that the CLT holds not just for symmetric binary random variables, but for any iid random variables $D_{i}$ symmetric about 0 . The steps consist of: 1 ) conditioning on $\left|d_{i}\right|, i=1, \ldots, n$, which creates independent, symmetric binary random variables; 2) using the uncunx applet to conclude that conditioned on the $\left|d_{i}\right|, S_{n}$ is approximately $\mathrm{N}\left(0, \sum_{i=1}^{n} d_{i}^{2}\right)$, or equivalently, $Z_{n}=S_{n} /\left(\sum_{i=1}^{n} d_{i}^{2}\right)^{1 / 2}$ is approximately standard normal; 3) arguing that because the conditional distribution of $Z_{n}$ given $\left|d_{i}\right|, i=1, \ldots, n$ is approximately standard normal for all possible values $\left|d_{1}\right|,\left|d_{2}\right| \ldots,\left|d_{n}\right|, \ldots$ (except those in a set of probability 0 ), $Z_{n}$ is unconditionally asymptotically standard normal as well; 4) arguing that $\hat{\sigma}^{2}=\sum D_{i}^{2} / n$ is very close to $\mathrm{E}\left(D_{1}^{2}\right)=\sigma^{2}$, so because $\sum D_{i} /\left(\sum D_{i}^{2}\right)^{1 / 2}=n^{1 / 2} S_{n} / \hat{\sigma}$ is approximately standard normal, so is $n^{1 / 2} S_{n} / \sigma$. From this argument the student can see that the CLT should hold whenever the iid random variables 
have a symmetric distribution with mean 0 and finite variance.

\section{Random Number Generation}

Thus far we have considered random variables symmetric about 0 , but an interesting modification of the quincunx allows us to visualize the inverse probability transform for generating random variables with an arbitrary continuous distribution $F$. In the first row, place the nail at the median of $F$. The 2 nails in the second row are placed at the first and third quartiles, while the 4 nails in the third row are at percentiles corresponding to probabilities $1 / 8,3 / 8$, $5 / 8$, and $7 / 8$, and similarly for subsequent rows. When a ball strikes the nail in the first row, it deflects either to the left or right, striking either the first or second nail in the second row. The size of the left deflection may not be the same as the size of the right deflection. Similarly, if the ball deflects left at the first row, hitting the nail at the $1 / 4$ quantile of the second row, it deflects left or right and strikes either the $1 / 8$ or $3 / 8$ quantile of the third row, etc. (Figure 5). The distribution of balls at the bottom of this uncunx with a large number of rows will be arbitrarily close to the density $f$ of $F$.

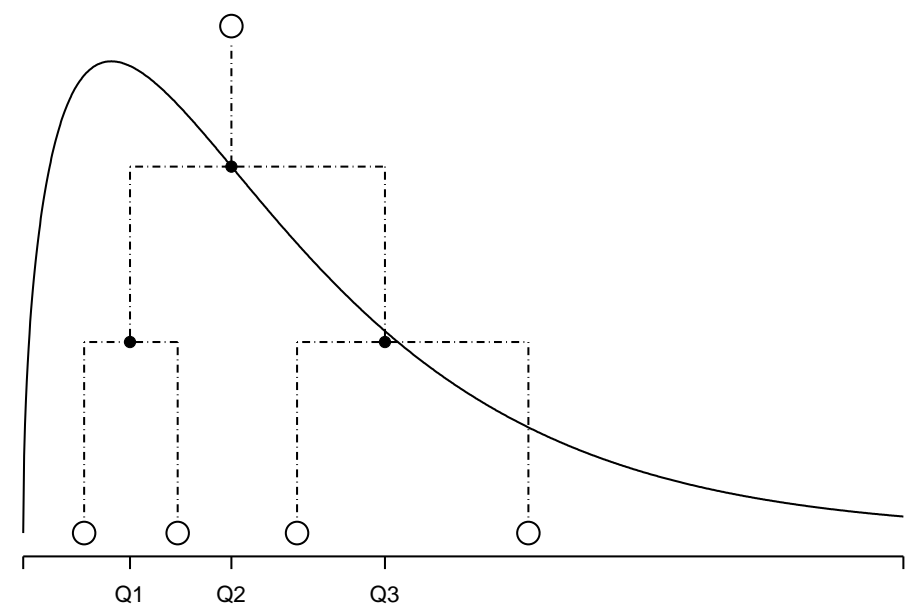

Figure 5: Using an uncunx to simulate from an arbitrary distribution. The nail in the first row divides the distribution into less than or greater than the median. The nail in the second row subdivides into below or above the quartiles Q1 and Q3. As we add rows, the distribution of balls at the bottom approaches that of the continuous curve. 
The applet can be used to illustrate this process for four different distributions: gamma $(2,1)$, gamma $(4,1)$, exponential, and lognormal. Click on the display and type "q" for quantile, which illustrates generation from a gamma $(2,1)$ distribution (pressing "q" repeatedly cycles through the other three distributions and returns to normal mode). The first row corresponds to the largest deflection because for the gamma $(2,1)$ distribution the distance between the median and the first or third quartile is larger than distances between subsequent quartiles and octiles, etc. The triangles get smaller as we move down the rows. Because the left-right deflections are of different sizes, the triangles are asymmetric. After sufficiently many balls have passed through the uncunx, the histogram strongly resembles the density $f(x)$ (Figure 6).

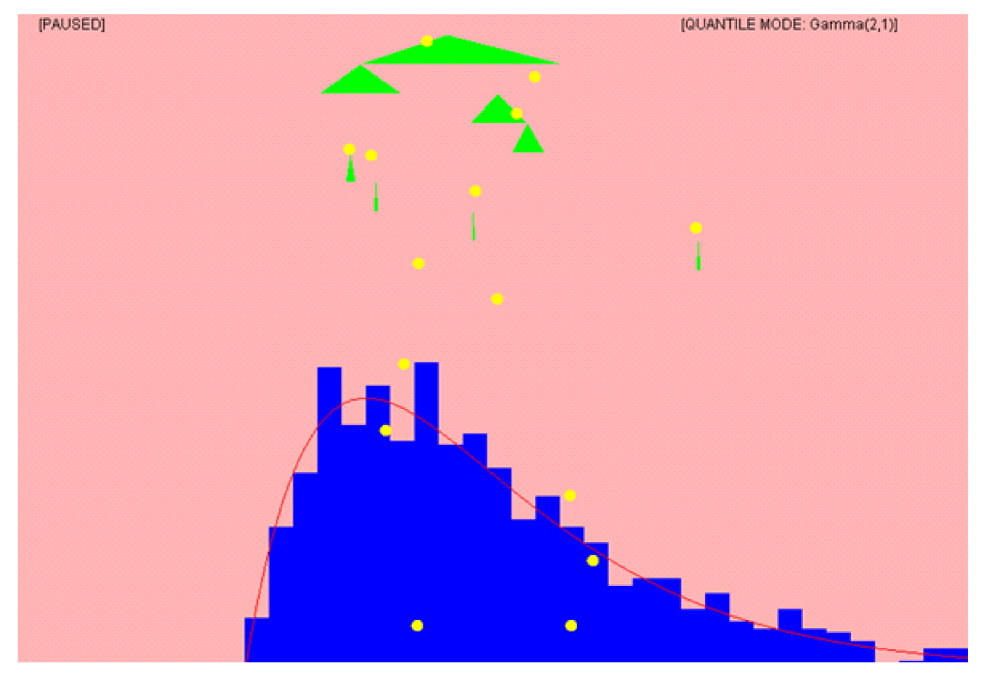

Figure 6: Using the applet to illustrate simulation from an arbitrary distribution with density $f(x)$ using the method described in the legend of Figure 5. The resulting histogram strongly resembles the density $f(x)$.

To see the connection between the applet and the generation of an arbitrary random deviate from a uniform deviate, imagine placing the nails not at the quantiles of the distribution, but at their corresponding probabilities. That is, the nail in the first row is placed at horizontal location $1 / 2$, the two nails in the second row are placed at horizontal locations $1 / 4$ and $3 / 4$, etc. Call this the probability uncunx as opposed to the quantile uncunx generated by the applet in quantile mode. There is exactly one path leading to each of the two nails in row 2 of the probability uncunx, exactly one path leading to each of the 4 nails in row 3 , 
and more generally, exactly one path leading to each of the $2^{i-1}$ nails in row $i$. Therefore, the distribution of balls in row $i$ is a discrete uniform on $1, \ldots, 2^{i-1}$. Take the same set of left-right deflections generated by the quantile uncunx and superimpose them on the probability uncunx. As the number of rows increases, the quantile uncunx generates a deviate from a distribution that is arbitrarily close to $F$, while the probability uncunx generates a deviate from a distribution that is arbitrarily close to a uniform $[0,1]$. Therefore, there is a 1-1 connection between generating a uniform deviate and generating an arbitrary continuous random variable. The process maps the percentiles of an arbitrary distribution to the corresponding percentiles of a uniform. The uncunx therefore gives a visual representation of the well-known result that one can simulate from any continuous distribution function $F$ by $F^{-1}(U)$, where $U$ is uniformly distributed (see, for example, page 156 of DeGroot, 1986).

The uncunx applet in quantile mode is interesting because the indicators of whether the deflections are left or right are iid Bernoullis, but the deflection sizes are not independent. For example, knowing that the first deflection was to the right pins down the deflection size in the second row to one of two choices; if the first row deflection had instead been to the left, the deflection size choices in the second row would have been different.

\section{Summary}

The quincunx allowed early statisticians to perform simulations long before the advent of modern computers. The problem is that building even a simple physical quincunx is difficult, and building one that incorporates the modifications in this paper is nearly impossible. Fortunately, the URL (1) provides a virtual universal quincunx that can be used to demonstrate a multitude of useful concepts, including the central limit theorem for independent but not identically distributed random variables, permutation tests in a paired setting, and the generation of a random variable with an arbitrary continuous distribution from a uniform variate. Young students are often enthralled by video games, so the uncunx applet may provide that spark that sways them to go into the field of statistics. Its versatility makes the uncunx an essential tool in mathematics and statistics classes of all levels. 


\section{Acknowledgment}

We would like to thank the referees, editor, and associate editor for suggestions that improved the paper markedly, and Wendy Lou, who introduced us to each other.

\section{References}

1. DeGroot, M.H. (1986). Probability and Statistics, 2nd ed. Addison Wesley, Reading Massachusetts.

2. Feller, W. (1957). An Introduction to Probability Theory and Its Applications, Vol 1. John Wiley and Sons, New York.

3. Good, P. (2005). Permutation, Parametric, and Bootstrap Tests of Hypotheses, 3rd ed. Springer, New York.

4. Kunert, J., Montag, A., and Pohlmann, S. (2001). The quincunx: history and mathematics. Statistical Papers 42, 143-169.

5. Parzen, E. (1960). Modern Probability Theory and Its Applications. John Wiley and Sons, New York.

6. Proschan, M.A. (2008). The normal approximation to the binomial. The American Statistician 62, 62-63.

7. Stigler, S.M. (1986). The History of Statistics: The Measurement of Uncertainty before 1900. The Belknap Press of Harvard University Press. Cambridge, Massachusetts.

8. van der Vaart, A.W. (1998). Asymptotic Statistics. Cambridge University Press. 